\title{
VARIABLE STRUCTURE OBSERVER DESIGN FOR A CLASS OF UNCERTAIN SYSTEMS WITH A TIME-VARYING DELAY
}

\author{
WEN-JENG LIU \\ Department of Electronic Engineering \\ Nan-Kai University of Technology, Tsao-tun 542, Nan-Tou, Taiwan, ROC \\ e-mail: wjliuankut. edu. tw
}

\begin{abstract}
Design of a state observer is an important issue in control systems and signal processing. It is well known that it is difficult to obtain the desired properties of state feedback control if some or all of the system states cannot be directly measured. Moreover, the existence of a lumped perturbation and/or a time delay usually reduces the system performance or even produces an instability in the closed-loop system. Therefore, in this paper, a new Variable Structure Observer (VSO) is proposed for a class of uncertain systems subjected to a time varying delay and a lumped perturbation. Based on the strictly positive real concept, the stability of the equivalent error system is verified. Based on the generalized matrix inverse approach, the global reaching condition of the sliding mode of the error system is guaranteed. Also, the proposed variable structure observer will be shown to possess the invariance property in relation to the lumped perturbation, as the traditional variable structure controller does. Furthermore, two illustrative examples with a series of computer simulation studies are given to demonstrate the effectiveness of the proposed design method.
\end{abstract}

Keywords: variable structure observer, invariance property, generalized matrix inverse approach.

\section{Introduction}

Lumped perturbations and time delays existing in many real-world systems, and the study of a class of uncertain systems subjected to time varying delays have become an interesting issue in recent years (Liu, 2005; Xiang et al., 2010; Hua et al., 2008; Shyu et al., 2005; Sun et al., 2008). The time delay phenomenon is quite commonly found in various systems, such as hydraulic/pneumatic, interconnected, communication, biological and long transmission ones. On the other hand, lumped perturbation can be a combination of system uncertainties, nonlinearities and/or external disturbances. In fact, the existence of a time delay and the presence of a lumped perturbation usually degrade the control performance or, even worse, produce an instability of the closed-loop system. Hence, the existence of a time delay and a lumped perturbation always renders the control problem much more complex and difficult.

In this paper, we consider an uncertain system with a time-varying delay whose dynamics are described by

$$
\begin{aligned}
\dot{x}(t)= & (A+\Delta A) x(t)+\left(A_{d}+\Delta A_{d}\right) x(t-\tau) \\
& +(B+\Delta B) u(t)+D w(t),
\end{aligned}
$$

$$
x(t)=\theta(t) \quad \text { for }-\tau \leq t<0,
$$

together with the output equation

$$
y(t)=C x(t),
$$

where $x(t) \in \mathbb{R}^{n}, u(t) \in \mathbb{R}^{m}$ and $y(t) \in \mathbb{R}^{h}$ denote the state variables, control inputs and measured outputs of the uncertain system, respectively. The nonzero timevarying delay is denoted by $\tau$. The function $\theta(t)$ represents a continuous vector-valued initial function. The matrices $A \in \mathbb{R}^{n \times n}, A_{d} \in \mathbb{R}^{n \times n}, B \in \mathbb{R}^{n \times m}$ and $C \in \mathbb{R}^{h \times n}$ are the nominal state matrix, the state-delay term matrix, the input matrix and the output matrix of the uncertain dynamic system, respectively. The symbols $\Delta A, \Delta A_{d}$ and $\Delta B$ are the uncertainty matrices. The function $w(t) \in \mathbb{R}^{n}$ denotes the nonlinear parts and/or the external disturbances of the system. The matrix $D \in \mathbb{R}^{n \times n}$ describes the influence of $w(t)$ on the system.

To control an uncertain system, it has been shown that Variable Structure Control (VSC) is a very effective method (DeCarlo et al., 1988; Hung et al., 1993). As is well known, in the VSC system, the trajectories of the closed-loop system will be forced onto a specified surface, which is the so-called sliding surface, and the trajectories will be kept on the sliding surface thereafter. Such 
a motion is referred to as the sliding mode (Utkin, 1992). During the sliding mode, the system remains insensitive to internal parameter variations and external disturbance. Moreover, when a sliding mode is achieved, the system will experience a reduced-order motion.

To deal with the effects arising from the existence of a time delay and uncertainties, the state feedback method is frequently used in system control design. Liu (2005) investigated the issue of robust asymptotic stabilization for uncertain time-delay systems with saturating actuators. Delay-dependent criteria for robust stabilization via linear state feedback were obtained. Xiang et al. (2010) dealt with the problem of fault tolerant control of a class of uncertain switched nonlinear systems with time delay under asynchronous switching. Hua et al. (2008) studied a state feedback control problem for a class of nonlinear time-delayed systems with matched uncertainties. The state transformation is needed in their design approach, but it may change an original state into another one with a different physical meaning in the new coordinates. Moreover, finding a suitable transformation or indirect estimated states may become another problem. Shyu et al. (2005) developed a new robust control law to stabilize an uncertain large-scale time delayed system with a dead-zone input via the state feedback method.

All of their discussions of such a problem were based on the assumption that all the system states are known. However, in practice, some or all of the system states cannot be directly measured. This fact becomes a barrier in acquiring the desired properties of state feedback control method. To overcome the problem of incomplete information of system states, the state observer could be an elegant solution to gain the desired data. State observer design is an important issue in control systems and signal processing. An observer is an auxiliary dynamic system for estimating the components of the original system state vectors. Luenberger (1971) first proposed the concept of the observer, and some significant advances have been made in observer theory (O'Reilly, 1983; Nijmeijer and Fossen, 1999). The so-called Luenberger observer can perform well when the system dynamics are known. However, if some uncertainties exist in the system, the estimation of the states may not be sufficiently accurate.

In addition to the Luenberger observer, other observer design techniques have been proposed. For example, Karafyllis and Kravaris (2007) studied the construction of observers for time-varying discrete-time systems and proposed some appropriate notions of robust complete observability. Liu (2004) proposed an algorithm for state observer design of two-dimensional linear shift-invariant systems via using the well-known one-dimensional system results. Röbenack and Lynch (2006) proposed two methods for nonlinear observer design which are based on a partial nonlinear observer canonical form. Moreover, Sliding Mode Observer (SMO) design of an uncer- tain system is an important issue of recent discussion. Slotine et al. (1987) examined the potential use of sliding surfaces for observer design for systems in companion form and in general form. Żak and Hui (1993) proposed a class of VSO for uncertain/nonlinear dynamic systems with bounded uncertainties, but they only discussed the sliding condition for the switching surface. Unel et al. (2008) used sliding mode observers to estimate the motion and the structure of a moving body with the aid of a change-coupled device camera. Yan and Edwards (2007) used an equivalent output error injection approach to consider the fault detection and estimation issues for a class of systems with uncertainty, and the fault estimation approach was dependent on the bounds on the uncertainty. Spurgeon (2008) presented an overview of both linear and non-linear SMO paradigms. Since their SMO design (Yan and Edwards, 2007; Spurgeon, 2008) is based on a state transformation, their results are indirectly obtained due to the requirement of coordinate transformation. Recently, using the generalized matrix inverse approach, Liu et al. (2009) proposed a new SMO for a class of uncertain systems. However, none of the above SMO design considered the state delay factor. It is worth emphasizing that the existence of time delay usually degrades the control performance or, even worse, causes instability in the closedloop system. Therefore, it is necessary to develop a new variable structure observer to deal with the state estimation problem for uncertain time delayed systems.

In this paper, utilizing the results derived by Liu et al. (2009) and VSC theory, the design of a new VSO for a class of uncertain systems with time-varying delay is accomplished. The proposed variable structure observer will be shown to possess the invariance property in relation to the lumped perturbation as the traditional variable structure controller does. Moreover, based on the strictly positive real concept, the stability of the equivalent error system is verified. Following the generalized matrix inverse approach, the global reaching condition of the sliding mode of the error system is guaranteed. Since the proposed VSO is not based on the transformed system model, it is no longer necessary to worry about the problems of finding a suitable transformation or indirect estimated states. As a consequence, the proposed VSO synthesizing procedure is simpler than the aforementioned VSO design found in the literature. Finally, some simulation results are illustrated to show the proposed variable structure observer is superior to the traditional one, designed without considering the time-varying delay term.

The rest of the paper is organized as follows. System and problem descriptions are introduced in Section 2. Section 3 derives the main results of the paper, which show how to construct the developed VSO. Section 4 verifies the proposed design method through two simulation studies. Finally, some concluding remarks are outlined in Section 5. 


\section{System and problem description}

In this paper, a class of uncertain time-varying delay systems whose dynamics as shown in (1)-(3) is considered. For the developed uncertain time-varying delay system, the pair $(A, B)$ is assumed to be controllable, and the pair $(A, C)$ observable. The following matching conditions are also assumed to hold:

$$
\begin{aligned}
\Delta A & =B E C, & \|E\| & \leq k_{1}, \\
\Delta A_{d} & =B F C, & \|F\| & \leq k_{2}, \\
\Delta B & =B G, & \|G\| & \leq k_{3}, \\
D w(t) & =B w_{m}(t), & \left\|w_{m}(t)\right\| & \leq k_{4},
\end{aligned}
$$

where $E, F, G$ are constant matrices, $w_{m}(t)$ is a vector with dimension $m . \quad k_{i}, i=1,2,3,4$, are known nonnegative constants, and $\|(\cdot)\|$ denotes the Euclidean norm when $(\cdot)$ is a vector, or the induced norm when $(\cdot)$ is a matrix. Further, it is well known that observable $(A, C)$ implies that one can find a matrix $K \in \mathbb{R}^{n \times h}$, such that the matrix

$$
\underline{A}=A-K C
$$

is Hurwitz.

Remark 1. Note that when the above matching conditions are satisfied, a lumped perturbation will only affect the initial conditions of the sliding mode equations. The state dynamics are insensitive to the lumped perturbation after the switching surfaces are hit.

Remark 2. Based on the matching condition (4), the uncertain time delayed system (1) can be rewritten as

$$
\dot{x}(t)=A x(t)+A_{d} x_{d}(t)+B[u(t)+f(t)],
$$

where

$$
\begin{aligned}
x_{d}(t) & =x(t-\tau), \\
f(t) & =E y(t)+F y_{d}(t)+G u(t)+w_{m}(t)
\end{aligned}
$$

and $f(t)$ represents a lumped perturbation which satisfies

$$
\|f(t)\| \leq k_{1}\|y\|+k_{2}\left\|y_{d}\right\|+k_{3}\|u\|+k_{4},
$$

where $y_{d}$ denotes the delay output.

Let the state estimate $x(t)$ be denoted by $\hat{x}(t)$, and the error difference between the estimate state and the true state by $e(t)$, i.e.,

$$
e(t)=x(t)-\hat{x}(t)
$$

Here, a variable structure observer under consideration is of the form

$$
\begin{aligned}
\dot{\hat{x}}(t)= & A \hat{x}(t)+B u(t) \\
& +K[y(t)-C \hat{x}(t)]+B N(t),
\end{aligned}
$$

where $K$ is a gain matrix designed as in (5) and the nonlinear switching term $N(t)$ will be used to ensure robustness against the lumped perturbation of the system (6) which will be designed later.

Subtracting (11) from (6), the governing error dynamics will be of the form

$$
\dot{e}(t)=(A-K C) e(t)+A_{d} x_{d}(t)+B(f-N(t)) .
$$

The goal of this paper is to design the gain matrix $K$ and the switching term $N(t)$, such that the uncertain error system with the time-varying delay (12) is globally asymptotically stable.

In the following section, we present schematic procedures of VSO design for a class of uncertain systems with time-varying delay. Usually, VSO design is a twophase process. Phase 1 is to choose a proper switching surface for the uncertain system such that the sliding motion on the switching surface possesses the desired properties. Phase 2 is to determine a switching term which is able to force the system to move toward the sliding surface in spite of the presence of uncertainties and then stay on it thereafter.

\section{Variable structure observer design}

At the beginning, a proper switching surface for the uncertain system is chosen such that the sliding motion on the switching surface possesses the desired properties. The switching surface is chosen as follows:

$$
\sigma(t)=S e(t)=M C e(t)=M(y-\hat{y}),
$$

where $\sigma(t) \in \mathbb{R}^{m}$ is a vector function, $S \in \mathbb{R}^{m \times n}, M \in$ $\mathbb{R}^{m \times h}$ are constant matrices and $m<n$. Assume that the product matrix $S B$ is nonsingular, and $S$ has full row rank.

A brief description for the strictly positive real concept is summarized (Khalil, 1996) for the stability of the equivalent error dynamics.

Definition 1. A transfer function $T(q)$ is positive real if $\operatorname{Re}[T(q)] \geq 0$ for all $\operatorname{Re}[q] \geq 0$. It is strictly positive real if $T(q-\varepsilon)$ is positive real for some $\varepsilon>0$, where $q$ is a complex number.

The following lemma is modified after Walcott and Żak (1987) as well as Khalil (1996), and will be used to verify the motion of the uncertain delayed error system on the switching surface to possess the property of insensitivity to the lumped perturbation.

Lemma 1. Let $T(q)=M C(q I-\underline{A})^{-1} B$ be an $m \times m$ transfer function matrix, where $\underline{A}$ is stable while $(\underline{A}, B, M C)$ is controllable and observable. Then $T(q)$ is strictly positive real if and only if there exist positive definite symmetric matrices $P$ and $W$ such that

$$
P \underline{A}+\underline{A}^{T} P=-W,
$$


where $(\cdot)^{T}$ denotes the transpose of $(\cdot)$.

It is well known that, when the governing error system operates on the sliding mode, the following conditions are satisfied:

$$
\sigma=0 \quad \text { and } \quad \dot{\sigma}=0
$$

Let $N_{e q}$ denote the equivalent control. From (12), (13) and (15) one gets

$$
\dot{\sigma}=S\left[(A-K C) e+A_{d} x_{d}+B f-B N_{e q}\right]=0 .
$$

Therefore, the equivalent control $N_{e q}$ is given as

$$
N_{e q}=(S B)^{-1} S\left[\underline{A} e+A_{d} x_{d}+B f\right] .
$$

Let $A_{d}=B L$, where $L$ is a constant matrix. Substituting (17) into (12), we can easily show that the equivalent error dynamics on the switching surface are in the form of

$$
\dot{e}_{e q}(t)=\left[I-B(S B)^{-1} S\right] \underline{A} e .
$$

Note that the resulting equivalent error dynamics (18) have to be asymptotically stable.

Remark 3. Based on Lemma 1, the involved system is required to be asymptotically stable and completely controllable. In other words, if the strictly positive real condition is satisfied, then $(\underline{A}, B)$ is controllable and the asymptotical stability of (18) can be guaranteed. Furthermore, from (18), we can see that Eqn. (18) is insensitive to lump uncertainty. In other words, the invariance property is also held for VSO design.

To ensure the existence of a sliding mode, the following Assumption 1 and Lemma 2 will be used.

Assumption 1. There exists a positive definite matrix $Q \in$ $\mathbb{R}^{m \times m}$, such that

$$
S \underline{A} S^{+}=-Q,
$$

where $S^{+}=S^{T}\left(S S^{T}\right)^{-1}$ denotes the Moore-Penrose inverse (generalized inverse) of $S$.

Lemma 2. (Utkin, 1992) The motion of the sliding mode (13) is asymptotically stable if the following condition is satisfied:

$$
\sigma^{T}(t) \dot{\sigma}(t)<0
$$

To satisfy the condition (20), the switching term $N(t)$ is designed as follows:

$$
\begin{aligned}
N(t)= & \eta \frac{\left(\sigma^{T} S B\right)^{T}}{\left\|\sigma^{T} S B\right\|}\left[k_{1}\|y\|+\left(\left\|L C^{+}\right\|+k_{2}\right)\left\|y_{d}\right\|\right. \\
& \left.+k_{3}\|u\|+k_{4}\right], \quad \eta>1 .
\end{aligned}
$$

Now, we are in a position to show that the proposed switching term can achieve the condition (20).

Theorem 1. Consider the uncertain error delayed system (12). If the switching term in (21) is applied to the error dynamic system (12), then the sliding mode and the attractiveness to the sliding surface (13) will be ensured.

Proof. Let the Lyapunov function candidate be in the form of

$$
V(t)=\frac{1}{2} \sigma^{T}(t) \sigma(t)
$$

Taking the time derivative of the Lyapunov function candidate yields

$$
\begin{aligned}
\dot{V}(t) & =\sigma^{T}(t) \dot{\sigma}(t) \\
& =\sigma^{T} S\left[\underline{A} e+A_{d} x_{d}+B f-B N\right] .
\end{aligned}
$$

Note that there are unmeasurable states $e$ and $x_{d}$ in the above equation. The generalized matrix inverse is useful to avoid using the unmeasurable states in VSO design. Hence, we have $e=S^{+} \sigma$ and $x_{d}=C^{+} y_{d}$, where $S^{+}$is defined in (19) and $C^{+}=C^{T}\left(C C^{T}\right)^{-1}$ is the generalized right inverse of the matrix $C$. Applying (19) to the above equation, one has

$$
\begin{aligned}
\dot{V}(t)= & -\sigma^{T} Q \sigma+Q^{T} S B L C^{+} y_{d}+\sigma^{T} S B f \\
& -\sigma^{T} S B N .
\end{aligned}
$$

Using (9), (21) and the property $\|A B\| \leq\|A\|\|B\|$ yields

$$
\begin{aligned}
\dot{V}(t) \leq & \lambda_{\min }(Q)\|\sigma\|^{2}+(1-\eta)\left\|\sigma^{T} S B\right\|\left[k_{1}\|y\|\right. \\
& \left.+\left(\left\|L C^{+}\right\|+k_{2}\right)\left\|y_{d}\right\|+k_{3}\|u\|+k_{4}\right]<0,
\end{aligned}
$$

where $\lambda_{\min }(Q)$ denotes the minimum positive eigenvalue of $Q$. The sliding condition (20) stated in Lemma 2 $\dot{V}(t)<0$, ensures the attractiveness to the sliding surface, and the proof is complete.

\section{Illustrative examples}

In this section, two examples are presented to illustrate the effectiveness of the proposed VSO scheme. Example 1 is a Single-Input Single-Output (SISO) case, in which four different cases will be considered to show that the proposed VSO is superior to the traditional ones. Example 2 is a Multi-Input Multi-Output (MIMO) case with a more complex delay term.

Example 1. Here, an illustrative example is chosen from the literature on the subject. The purpose of this example is to demonstrate the effectiveness of the proposed VSO, and four different cases will be considered to show 
that the proposed VSO is superior to the traditional ones. The following dynamical system is adopted from Liu $e t$ al. (2009), Walcott and Żak (1987) as well as Żak and Hui (1993), in which all the parameters are the same as their parameters except the time-varying delay term. The observers with and without considering the time-varying delay terms were simulated on a computer.

Consider an uncertain system with a time-varying delay given by

$$
\begin{aligned}
\dot{x}(t)= & {\left[\begin{array}{ll}
0 & 1 \\
0 & 0
\end{array}\right] x(t)+\left[\begin{array}{cc}
0 & 0 \\
-1 & -1
\end{array}\right] x_{d}(t) } \\
& +\left[\begin{array}{l}
0 \\
1
\end{array}\right] u(t)+\left[\begin{array}{l}
0 \\
1
\end{array}\right] f(t)
\end{aligned}
$$

with the output equation

$$
y(t)=\left[\begin{array}{ll}
1 & 1
\end{array}\right] x(t),
$$

which are subjected to the lumped perturbation $f(t)=$ $-\sin \left(x_{1}(t)\right)$, and the time-varying delay $\tau=0.8|\cos (t)|$.

The purpose of designing a VSO with the structure (8) is to ensure that the uncertain time-varying delay error dynamics (10) are globally asymptotically stable. It can be easily checked that the nominal system $(A, C)$ is observable. Therefore, there exists a gain matrix $K=\left[\begin{array}{ll}1 & 1\end{array}\right]^{T}$, such that the eigenvalues of $\underline{A}=A-K C$ are $\{-1,-1\}$.

Now, the observer equation corresponding to the developed system is given by

$$
\begin{aligned}
{\left[\begin{array}{c}
\dot{\hat{x}}_{1} \\
\dot{\hat{x}}_{2}
\end{array}\right]=} & {\left[\begin{array}{cc}
-1 & 0 \\
-1 & -1
\end{array}\right]\left[\begin{array}{l}
\hat{x}_{1} \\
\hat{x}_{2}
\end{array}\right]+\left[\begin{array}{l}
0 \\
1
\end{array}\right] u } \\
& +\left[\begin{array}{l}
1 \\
1
\end{array}\right] y+\left[\begin{array}{l}
0 \\
1
\end{array}\right] N(t) .
\end{aligned}
$$

The switching surface is chosen as

$$
\sigma(t)=y-\hat{y} .
$$

In this simulation study, the initial values are chosen as $\left[x_{10} x_{20}\right]^{T}=[-11]^{T}$, and the following parameters are given for the computer simulation:

$$
\eta=1.2, \quad k_{1}=0.5, \quad k_{2}=0.5, \quad k_{3}=0, \quad k_{4}=1.5 .
$$

In order to provide a comparison with the existing literature, the following cases are considered:

Case 1. Applying the traditional VSO (Walcott and Żak, 1987) to the system (26) without considering the timevarying delay term, i.e., $A_{d}=0$.

Case 2. Applying the traditional VSO (Walcott and Żak, 1987) to the system (26) with considering the timevarying delay term, i.e., $A_{d} \neq 0$.

Case 3. Applying the proposed VSO to the system (26) without considering the time-varying delay term, i.e., $A_{d}=0$.
Case 4. Applying the proposed VSO to the system (26) with considering the time-varying delay term, i.e., $A_{d} \neq$ 0 .

Figures 1-4 show the time responses of system states and error states for Cases 1-4, respectively, which consist of (a) the system state $x_{1}(t)$ and the estimated state $\hat{x}_{1}(t)$, (b) the system state $x_{2}(t)$ and the estimated state $\hat{x}_{2}(t)$, and (c) the error states $e_{1}(t)$ and $e_{2}(t)$. Applying the traditional VSO derived by Walcott and Zak (1987) to the system without considering the state delayed term, one gets the state trajectory evolution shown in Fig. 1. However, for Case 2, when applying the traditional VSO derived by Walcott and Żak (1987) to the system with considering the state delayed term, it does not ensure the stability of the uncertain system with time-varying delay, and the poor performance is shown in Fig. 2. For Cases 3 and 4 , the system and error states are shown in Figs. 3 and 4, respectively.
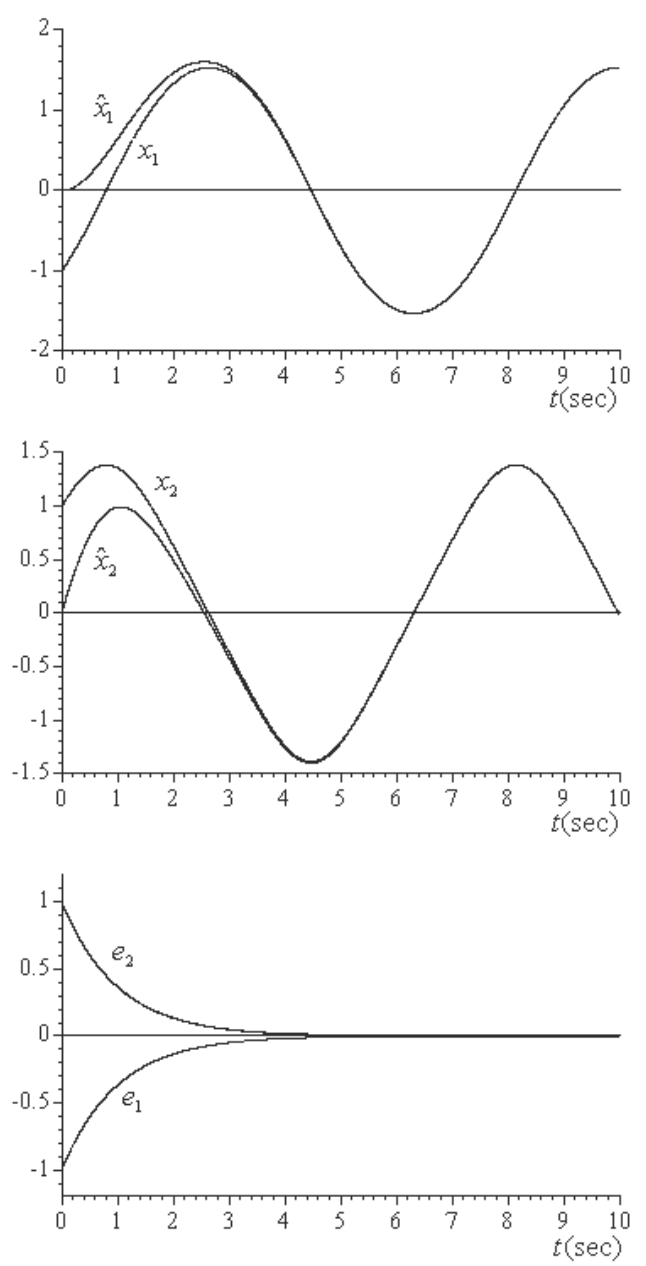

Fig. 1. SISO system without considering the time-varying delay term controlled by the traditional VSO (Walcott and Żak, 1987): $x_{1}(t)$ and $\hat{x}_{1}(t)(\mathrm{a}), x_{2}(t)$ and $\hat{x}_{2}(t)(\mathrm{b}), e_{1}(t)$ and $e_{2}(t)(\mathrm{c})$. 

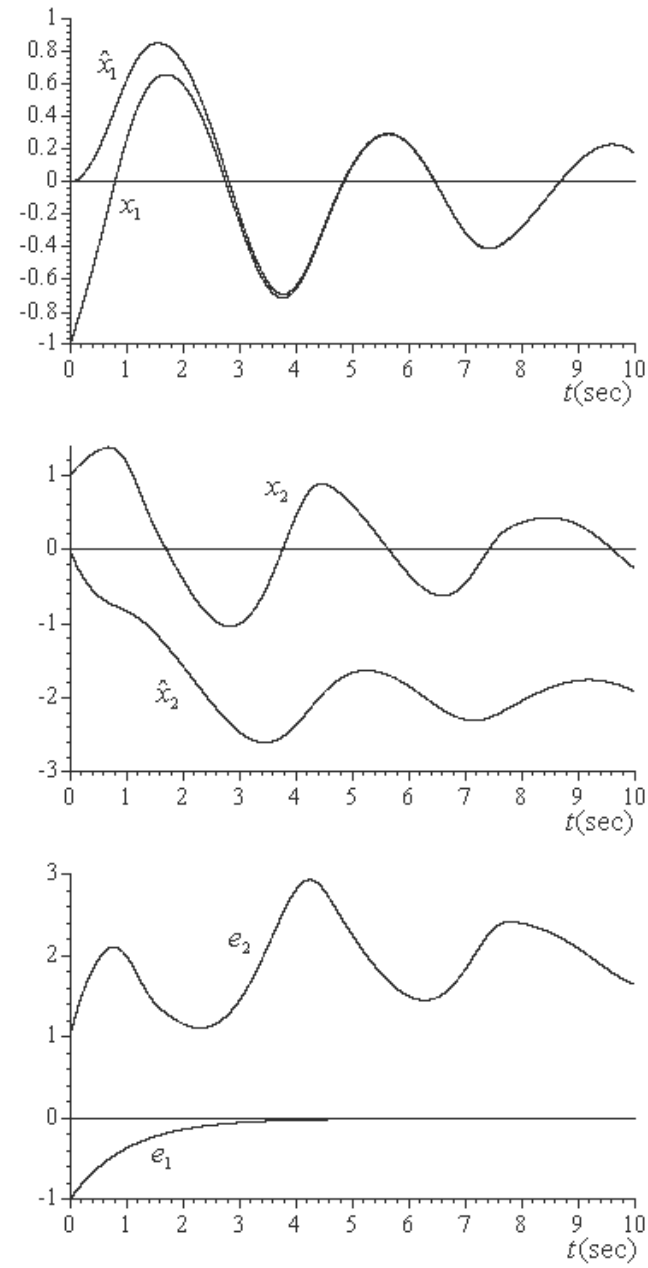

Fig. 2. SISO system with considering the time-varying delay term controlled by the traditional VSO (Walcott and Żak, 1987): $x_{1}(t)$ and $\hat{x}_{1}(t)(\mathrm{a}), x_{2}(t)$ and $\hat{x}_{2}(t)(\mathrm{b}), e_{1}(t)$ and $e_{2}(t)(\mathrm{c})$

From these simulation results, one can see that the proposed VSO law can work effectively for a class of uncertain systems with or without considering the timevarying delay. However, the traditional VSO is not applicable to the uncertain system with considering the timevarying delay term.

Example 2. In Example 1, a single-input single-output VSO is shown for comparison with the existing literature. Now, we consider the following uncertain time-varying delay systems with multiple inputs, multiple outputs and a more complex delay term:

$$
\dot{x}(t)=\left[\begin{array}{ccc}
1 & 0 & 0 \\
0 & -5 & 1 \\
2 & -3 & 1
\end{array}\right] x(t)+\left[\begin{array}{ccc}
-1 & 1 & -1 \\
1 & 0 & 1 \\
0 & 1 & 0
\end{array}\right] x_{d}(t)
$$
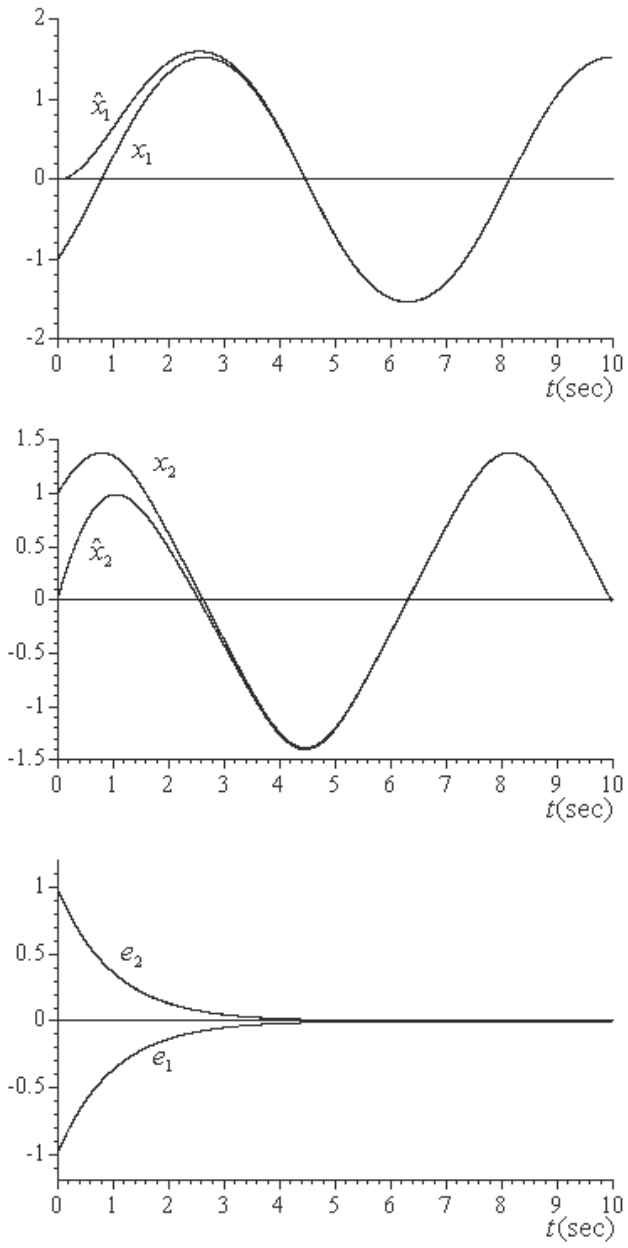

Fig. 3. SISO system without considering the time-varying delay term controlled by the proposed VSO: $x_{1}(t)$ and $\hat{x}_{1}(t)$ (a), $x_{2}(t)$ and $\hat{x}_{2}(t)(\mathrm{b}), e_{1}(t)$ and $e_{2}(t)(\mathrm{c})$.

$$
+\left[\begin{array}{cc}
-1 & 0 \\
1 & 1 \\
0 & 1
\end{array}\right] u(t)+\left[\begin{array}{cc}
-1 & 0 \\
1 & 1 \\
0 & 1
\end{array}\right] f(t)
$$

with the output equation

$$
y(t)=\left[\begin{array}{lll}
1 & 0 & 0 \\
0 & 0 & 1
\end{array}\right] x(t),
$$

where

$$
\begin{array}{rlrl}
\dot{x}(t) & =\left[\begin{array}{l}
\dot{x}_{1}(t) \\
\dot{x}_{2}(t) \\
\dot{x}_{3}(t)
\end{array}\right], & x(t)=\left[\begin{array}{l}
x_{1}(t) \\
x_{2}(t) \\
x_{3}(t)
\end{array}\right], \\
x_{d}(t)=\left[\begin{array}{l}
x_{1}(t-\tau) \\
x_{2}(t-\tau) \\
x_{3}(t-\tau)
\end{array}\right], & u(t)=\left[\begin{array}{l}
u_{1}(t) \\
u_{2}(t)
\end{array}\right], \\
y(t)=\left[\begin{array}{l}
y_{1}(t) \\
y_{2}(t)
\end{array}\right], & f(t)=\left[\begin{array}{l}
\sin \left(x_{1}\right) \\
\cos \left(x_{3}\right)
\end{array}\right]
\end{array}
$$

and the time-varying delay $\tau=0.5|\sin (t)|$. 

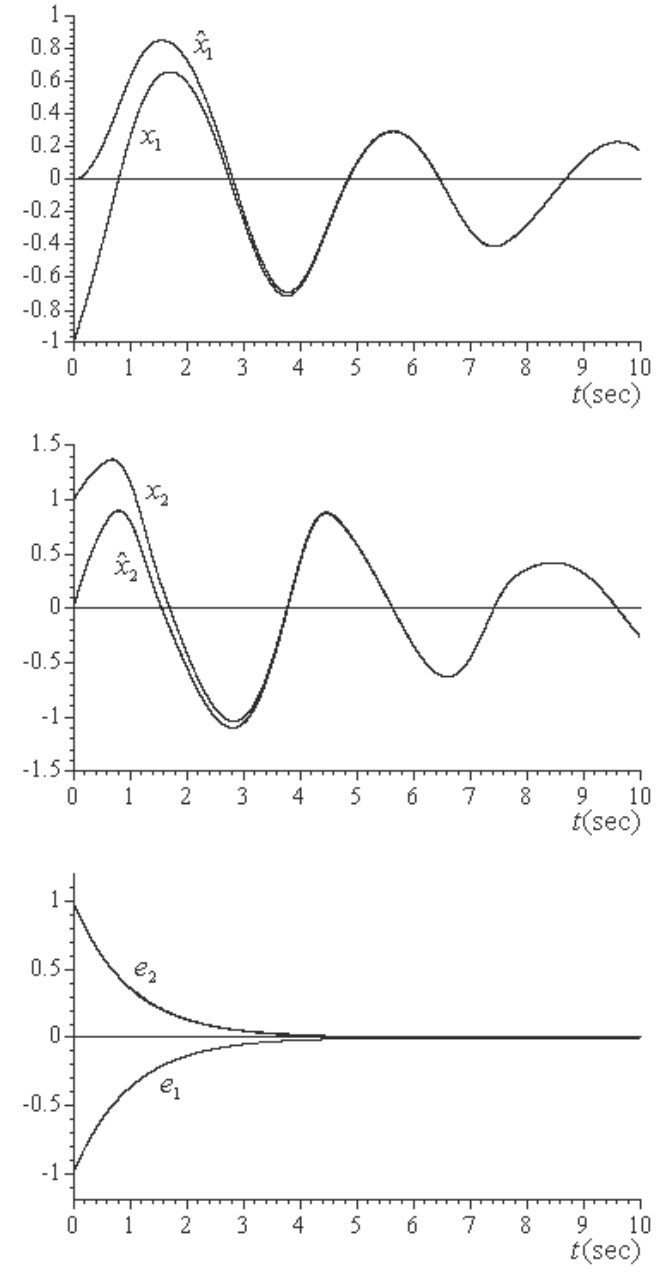

Fig. 4. SISO system with considering the time-varying delay term controlled by the proposed VSO: $x_{1}(t)$ and $\hat{x}_{1}(t)$ (a), $x_{2}(t)$ and $\hat{x}_{2}(t)(\mathrm{b}), e_{1}(t)$ and $e_{2}(t)(\mathrm{c})$.

One can easily check that the nominal system $(A, C)$ is observable, and hence a gain matrix $K$ can be found as follows:

$$
K=\left[\begin{array}{ll}
4 & 0 \\
0 & 1 \\
1 & 5
\end{array}\right]
$$

such that the eigenvalues of $\underline{A}=A-K C$ turn out to be $\{-3,-4,-5\}$.

From the system (11), we have that the VSO under consideration is of the form

$$
\begin{aligned}
\dot{x}(t)= & {\left[\begin{array}{ccc}
-3 & 0 & 0 \\
0 & -5 & 0 \\
1 & -3 & -4
\end{array}\right] \hat{x}(t)+\left[\begin{array}{cc}
-1 & 0 \\
1 & 1 \\
0 & 1
\end{array}\right] u(t) } \\
& +\left[\begin{array}{ll}
4 & 0 \\
0 & 1 \\
1 & 5
\end{array}\right] y(t)+\left[\begin{array}{cc}
-1 & 0 \\
1 & 1 \\
0 & 1
\end{array}\right] N(t),
\end{aligned}
$$

where

$$
\hat{x}(t)=\left[\begin{array}{c}
\hat{x}_{1}(t) \\
\hat{x}_{2}(t) \\
\hat{x}_{3}(t)
\end{array}\right], N(t)=\left[\begin{array}{c}
N_{1}(t) \\
N_{2}(t)
\end{array}\right] .
$$

The switching surfaces are chosen as

$$
\sigma_{1}(t)=y_{1}-\hat{y}_{1}, \quad \sigma_{2}(t)=y_{2}-\hat{y}_{2} .
$$

In this numerical simulation, the initial values are chosen as $\left[x_{10} x_{20} x_{30}\right]^{T}=\left[\begin{array}{lll}1 & -1 & 0.5\end{array}\right]^{T}$, and the following parameters are given for the computer simulation:

$\eta=1.2, \quad k_{1}=1.1, \quad k_{2}=1.2, \quad k_{3}=0, \quad k_{4}=1.5$.

From Eqn. (21), the switching terms are designed as follows:

$$
\begin{aligned}
N_{1}(t)= & 1.2 \frac{-\sigma_{1}}{\left\|\sigma^{T} S B\right\|}[1.1\|y\| \\
& \left.+(\sqrt{2}+1.2)\left\|y_{d}\right\|+1.5\right], \\
N_{2}(t)= & 1.2 \frac{-\sigma_{2}}{\left\|\sigma^{T} S B\right\|}[1.1\|y\| \\
& \left.+(\sqrt{2}+1.2)\left\|y_{d}\right\|+1.5\right] .
\end{aligned}
$$

Figure 5 shows the time responses of system states and estimated states as (a) $x_{1}(t)$ and $\hat{x}_{1}(t)$, (b) $x_{2}(t)$, and $\hat{x}_{2}(t)$ and (c) $x_{3}(t)$ and $\hat{x}_{3}(t)$. Furthermore, Fig. 6 shows the time responses of error states $e_{1}(t), e_{2}(t)$ and $e_{3}(t)$.

From these simulation results, it can be seen that the estimated states are all approaching the corresponding system states, and error states are all asymptotically stable, that is, the proposed VSO laws can work effectively for uncertain complex time-varying delay systems with multiple inputs, multiple outputs and with the presence of lumped perturbations.

\section{Conclusions}

Design of state observers is an important issue in control systems and signal processing. In this paper, we have made an attempt to extend the traditional Luenberger observer to generate a new VSO scheme, which is robust in the presence of lumped perturbations. The following main results have been obtained:

(i) It is unnecessary to design the proposed VSO through the transformed system model. As a consequence, the difficulty in finding a suitable transformation or the indirect estimated procedure no longer exists in the proposed observer design, and the proposed VSO synthesizing procedure is simpler than the VSO design presented in the literature.

(ii) Based on the generalized matrix inverse approach, the existence of a sliding mode and the attractiveness to the sliding surface are ensured. 

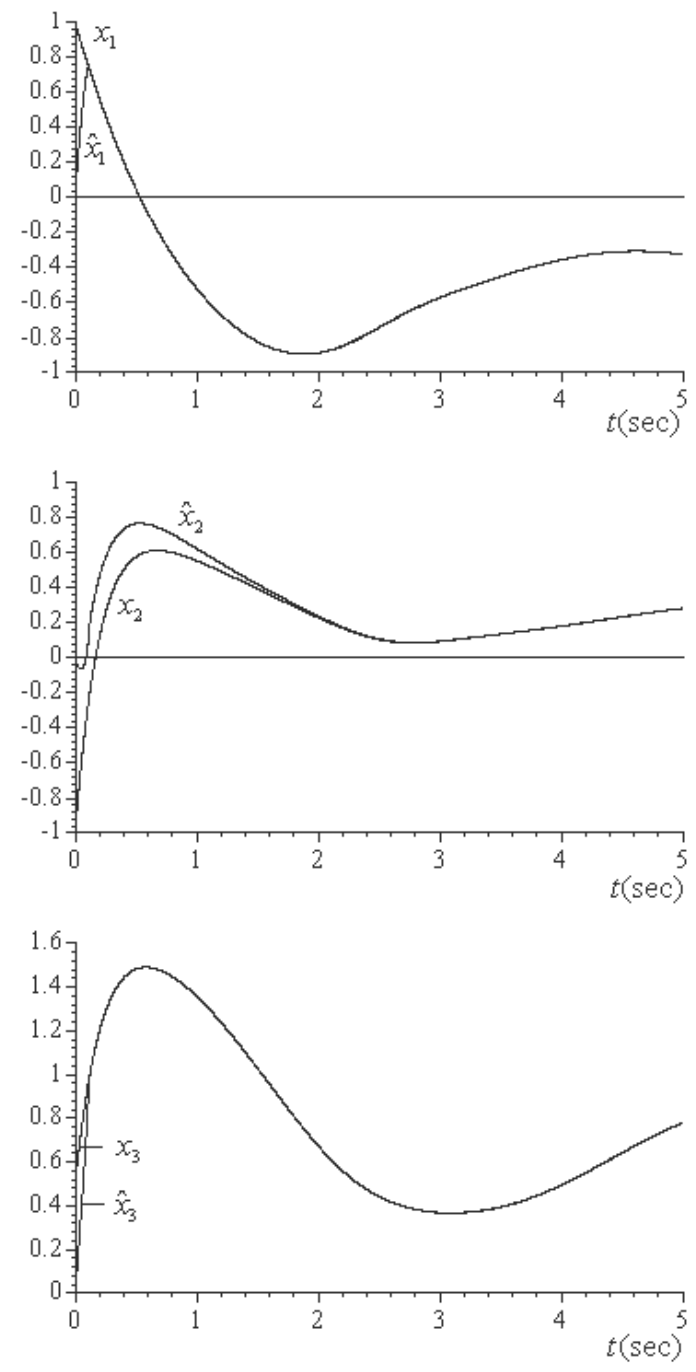

Fig. 5. Time responses of system states and estimated states in a MIMO system: $x_{1}(t)$ and $\hat{x}_{1}(t)(\mathrm{a}), x_{2}(t)$ and $\hat{x}_{2}(t)$ (b), $x_{3}(t)$ and $x_{3}(t)$ (c).

(iii) Using the strictly positive real concept, the stability of the equivalent error system has been demonstrated.

(iv) The proposed VSO bears the invariance property in relation to lumped perturbation as the traditional sliding mode controller does.

Furthermore, two illustrative examples with a series of computer simulation studies are given to demonstrate the effectiveness of the proposed design method.

It is expected that the proposed VSO design is applicable to many practical applications, such as electrical power systems, electromechanical systems, computer network systems, etc. Further, it is also expected that the proposed VSO approach can be extended to other more complex uncertain nonlinear systems, including a class of

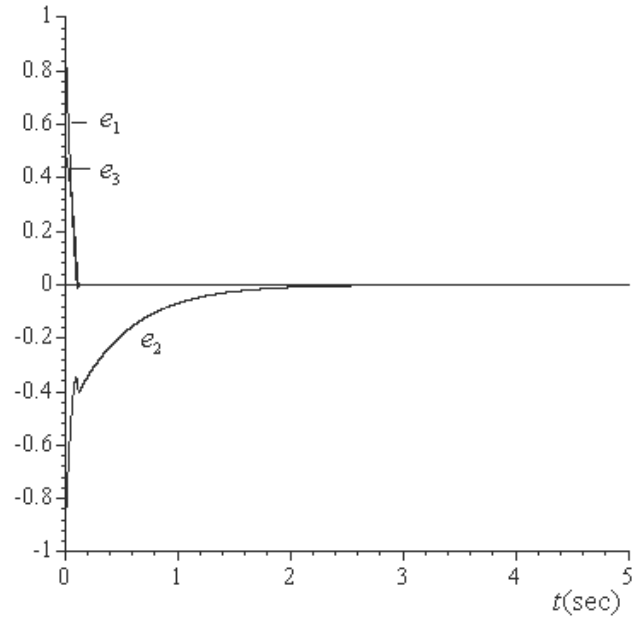

Fig. 6. Time responses of error states in a MIMO system: $e_{1}(t)$, $e_{2}(t)$ and $e_{3}(t)$

systems with saturation and/or dead-zone nonlinearity.

\section{Acknowledgment}

The author would like to thank the editors and the anonymous reviewers for their constructive comments that helped to improve the paper. This work was supported by the National Science Council of Taiwan under the contract NSC-101-2221-E-252-003-.

\section{References}

DeCarlo, R.A., Żak, S.H. and Matthews, G.P. (1988). Variable structure control of nonlinear multivariable systems: A tutorial, Proceedings of the IEEE 76(3): 212-232.

Hua, C., Wang, Q. and Guan, X. (2008). Memoryless state feedback controller design for time delay systems with matched uncertain nonlinearities, IEEE Transactions on Automatic Control 53(3): 801-807.

Hung, J.Y., Gao, W.B. and Hung, J.C. (1993). Variable structure control: A survey, IEEE Transactions on Industrial Electronic 40(1): 2-22.

Karafyllis I. and Kravaris, C. (2007). On the observer problem for discrete-time control systems, IEEE Transactions on Automatic Control 52(1): 12-25.

Khalil, H.K. (1996). Nonlinear Systems, Prentice-Hall, Upper Saddle River, NJ.

Liu, P.L. (2005). Delay-dependent asymptotic stabilization for uncertain time-delay systems with saturating actuators, International Journal of Applied Mathematics and Computer Science 15(1): 45-51.

Liu, W.J. (2004). Design of the observer feedback gain for twodimensional discrete systems, IEEE Signal Processing Letters 11(4): 413-415. 
Liu, W.J., Shyu, K.K. and Hsu, K.C. (2009). Sliding mode observer design for a class of uncertain systems, 2009 CACS International Automatic Control Conference, Taipei, Taiwan.

Luenberger, D. (1971). An introduction to observers, IEEE Transactions on Automatic Control 16(6): 596-602.

Nijmeijer, H. and Fossen, T.I.(1999). New Directions in Nonlinear Observer Design, Springer-Verlag, London.

O'Reilly, J. (1983). Observer for Linear Systems, Academic Press, New York, NY.

Röbenack, K. and Lynch, A.F. (2006). Observer design using a partial nonlinear observer canonical form, International Journal of Applied Mathematics and Computer Science 16(3): 333-343.

Shyu, K.K., Liu, W.J. and Hsu, K.C. (2005). Design of largescale time-delayed systems with dead-zone input via variable structure control, Automatica 41(7): 1239-1246.

Slotine, J.J.E., Hedrick, J.K. and Misawa, E.A. (1987). On sliding observers for non-linear systems, ASME Journal of Dynamic Systems, Measurement, and Control 109: 245-252.

Spurgeon, S.K. (2008). Sliding mode observers: A survey, International Journal of Systems Science 39(8): 751-764.

Sun, X.M., Wang, W., Liu, G.P. and Zhao, J. (2008). Stability analysis for linear switched systems with time-varying delay, IEEE Transactions on Systems, Man, and Cybernetics. Part B: Cybernetics, 38(2): 528-533.

Unel, M., Sabanovic, A., Yilmaz, B. and Dogan, E. (2008). Visual motion and structure estimation using sliding mode observers, International Journal of Systems Science 39(2): 149-161.

Utkin, V.I. (1992). Sliding Modes in Control and Optimization, Springer-Verlag, Berlin/Heidelberg.
Walcott, B.L. and Żak, S.H. (1987). State observation of nonlinear uncertain dynamical systems, IEEE Transactions on Automatic Control 32(2): 166-170.

Xiang, Z., Wang, R. and Chen, Q. (2010). Fault tolerant control of switched nonlinear systems with time delay under asynchronous switching, International Journal of Applied Mathematics and Computer Science 20(3): 497-506, DOI: 10.2478/v10006-010-0036-0.

Yan, X.G. and Edwards, C. (2007). Nonlinear robust fault reconstruction and estimation using a sliding mode observer, Automatica 43(9): 1605-1614.

Żak, S.H. and Hui, S. (1993). Output feedback variable structure controllers and state estimators for uncertain/nonlinear dynamic systems, IEE Proceedings D 140(2): 41-50.

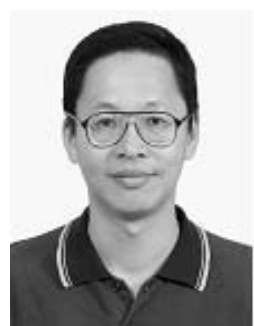

Wen-Jeng Liu received the B.Sc. degree in electrical engineering, the M.Sc. degree in computer science and information engineering, and the Ph.D. degree in electrical engineering, all from the National Central University, Chung-Li, Taiwan, ROC. He has been with the Electronics Research and Service Organization (ERSO), Industrial Technology Research Institute, Hsinchu, Taiwan. He has also been a design engineer with the Chung-Shan Institute of Science and Technology, Taoyuan, Taiwan. He is now an associate professor with the Department of Electronic Engineering, Nan Kai University of Technology, Nantou, Taiwan. His research interests include variable structure systems, sliding mode control, large-scale systems, time delayed systems, non-linear systems and uncertain systems.

Received: 11 November 2010 Revised: 12 May 2011

Re-revised: 22 October 2011 